\title{
Application of the World Health Organization Quality of Life Instrument, Short Form (WHOQOL-BREF) to patients with cataract
}

\author{
Ali Gholami ${ }^{1,2}$, Mahmood Tavakoli Araghi ${ }^{3}$, Fatemeh Shamsabadi ${ }^{3}$, Mahdiye Bayat $^{3}$, \\ Fatemeh Dabirkhani ${ }^{3}$, Farhad Moradpour ${ }^{4}$, Kamyar Mansori ${ }^{5}$, Yousef Moradi ${ }^{6}$, Abdolhalim Rajabi $^{2}$ \\ ${ }^{1}$ Department of Public Health, School of Public Health, Neyshabur University of Medical Sciences, Neyshabur; '²Department of Epidemiology, \\ School of Public Health, Iran University of Medical Sciences, Tehran; ${ }^{3}$ Student Research Committee, Neyshabur University of Medical \\ Sciences, Neyshabur; ${ }^{4}$ Social Determinants of Health Research Center, Kurdistan University of Medical Sciences, Sanandaj; ${ }^{5}$ Department of \\ Epidemiology, School of Public Health, Shahid Beheshti University of Medical Sciences, Tehran; ${ }^{6}$ Pars Advanced and Minimally Invasive \\ Manners Research Center, Pars Hospital, Iran University of Medical Sciences, Tehran, Iran
}

OBJECTIVES: Cataract is a prevalent disease in the elderly, and negatively influences patients' quality of life. This study was conducted to study the application of the World Health Organization Quality of Life Instrument, Short Form (WHOQOL-BREF) to patients with cataract.

METHODS: In this cross-sectional study, 300 patients with cataract were studied in Neyshabur, Iran from July to October 2014. The Iranian version of the WHOQOL-BREF questionnaire was used to measure their quality of life. Cronbach's alpha coefficient, Pearson's correlation coefficient, the paired t-test, the independent t-test, and a linear regression model were used to analyze the data in SPSS version 16.0 (SPSS Inc., Chicago, IL, USA).

RESULTS: The mean age of the participants was $68.11 \pm 11.98$ years, and most were female $(53 \%)$. The overall observed Cronbach's alpha coefficient for the WHOQOL-BREF was 0.889 , ranging from 0.714 to 0.810 in its four domains. The total mean score of the respondents on the WHOQOL-BREF was 13.19. The highest and lowest mean scores were observed in the social relationship domain (14.11) and the physical health domain (12.29), respectively. A backward multiple linear regression model found that duration of disease and marital status were associated with total WHOQOL scores, while age, duration of disease, marital status, and income level were associated with domains one through four, respectively $(\mathrm{p}<0.05)$.

CONCLUSIONS: The reliability analysis conducted in this study indicated that the WHOQOL-BREF scale exhibited an acceptable degree of internal consistency in the measurement of the quality of life of patients with cataract. It was also found that the patients with cataract who were surveyed reported a relatively moderate quality of life.

KEY WORDS: Cataract, Quality of life, World Health Organization, Reproducibility of results, Questionnaires, Cross-sectional study, Iran

Correspondence: Abdolhalim Rajabi
Department of Epidemiology, School of Public Health, Iran University of
Medical Sciences, Next to the Milad Tower, Hemmat Highway, Tehran
1449614535, Iran
Tel: +98-9106764431, Fax: +98-2188622707, E-mail: rajabiepid@gmail.com

Received: Jan 13, 2016, Accepted: Feb 4, 2016, Published: Feb 4, 2016

This article is available from: http://e-epih.org/

(C) 2016, Korean Society of Epidemiology

(CC) This is an open-access article distributed under the terms of the Creative Commons Attribution License (http://creativecommons.org/licenses/by/3.0/), which permits unrestricted use, distribution, and reproduction in any medium, provided the original work is properly cited.

\section{INTRODUCTION}

Cataract is a common disease in the elderly, and is especially prevalent in those older than 65 years. Cataract manifests as double or distorted vision, halo or glare vision, blurry vision, colors appearing differently, and gradual deterioration of the vision [1]. Surgical and non-surgical management are two strategies for treating cataract. The non-surgical strategy is typically performed during the early stages of cataract development, 
while the operative strategy is generally performed when a cataract affects patients' daily activities [2]. Snellen visual acuity has been widely used in clinical practice to measure a patient's visual function. Vision problems due to cataract have negative effects on patients' quality of life. Quality of life is defined by World Health Organization (WHO) as "an individual's perception of their position in life in the context of the culture and value systems in which they live and in relation to their goals, expectations, standards and concerns" [3]. The simple evaluation of the prevalence of cataract and its associated medical problems cannot convey the full meaning of its impact on the physical, mental, and social well-being of affected individuals. Quality of life measures allow for a more comprehensive understanding of the problems due to cataract. However, in order to study quality of life, we must be able to measure it. The World Health Organization Quality of Life Instrument, Short Form (WHOQOL-BREF) questionnaire is a commonly utilized generic measure of quality of life that is used to measure quality of life in healthy people and in different groups of patients [420]. The WHOQOL-BREF questionnaire is available in many languages, and has been translated into Persian and validated in Iran by Nedjat [21].Therefore, this study was conducted to study the utility of the WHOQOL-BREF in assessing the quality of life of patients with cataract.

\section{MATERIALS AND METHODS}

In this cross-sectional study, data were collected between July and October 2014 from all cataractous patients who received surgical treatment at two hospitals in the city of Neyshabur (northeastern Iran). All patients provided informed consent after being acquainted with the purpose of the study.

\section{Procedure and study instrument}

In this study, the questionnaires were filled out in personal interviews, but before the interviews, all participants were informed that their responses would remain confidential. The validated Persian-language version of the WHOQOL-BREF was used in this study. This questionnaire contains two items assessing overall quality of life and general health, as well as 24 other items divided into four domains: physical health (domain 1 ) with seven items, psychological health (domain 2) with six items, social relationships (domain 3) with three items, and environmental health (domain 4) with eight items. Each item is rated on a five-point Likert scale and scored from one to five on a response scale. According to the guidelines, the raw domain scores for the WHOQOL-BREF were transformed to a score between four and 20 [22]. The scores of each domain are scaled in a positive direction (i.e., lower scores denote lower quality of life).
The mean score of the items in each domain is used to calculate the domain scores, which are ultimately transformed linearly to a scale of zero to $100[23,24]$. The inclusion criteria applied in this study were: (1) the presence of cataract, (2) residence in Neyshabur, and (3) agreement to participate in the study.

\section{Dependent and independent variables}

In this study, the four domains of the WHOQOL-BREF questionnaire were considered dependent variables and other data (age, sex, education level, marital status, monthly income level, place of residence, and duration of disease) were considered independent variables. The age of the participants was represented by two categories: 65 years of age or younger and greater than 65 years of age. The education level of participants was classified as illiterate or literate. Marital status was categorized into two categories: single/divorced and married. Income level was divided into the two categories of $\leq \$ 170$ and $>\$ 170$ per month. The criterion of place of residence was categorized as urban and rural. The time interval from the detection of cataract until the time of the survey was divided into two categories: $\leq 30$ days and $>30$ days.

\section{Statistical analyses}

In this study, the data were analyzed using SPSS version 16.0 (SPSS Inc., Chicago, IL, USA). The descriptive analyses included frequencies, percentages, ranges, means, and standard deviations (SD). The reliability of the WHOQOL-BREF domains and overall quality of life were assessed using Cronbach's alpha, with scores of 0.70 and over deemed acceptable [25]. We used Pearson's correlation coefficient to determine the level of agreement between the four domains of the WHOQOL-BREF. The paired t-test was used to compare the mean scores of the different domains of the WHOQOL-BREF. The independent t-test and a linear regression model were used to investigate the relationship between patients' quality of life and their characteristics. Transformed scores were used for statistical analyses in all domains, and the level of significance was set at $\mathrm{p}<0.05$ for all analyses.

\section{RESULTS}

A total of 300 patients with cataract filled out the WHOQOL BREF questionnaire in this study. The characteristics of the study population are shown in Table 1. The mean age of the study population was $68.11 \pm 11.98$ years. Of the participants in this study, 158 (53\%) were female and $142(47 \%)$ were male. Table 2 presents the missing responses, mean score, SD, and floor and ceiling effects for each item. The highest and lowest mean scores were observed in the personal relationship (3.99) and leisure 
activity (2.09) items, respectively. The percentage of respondents scoring at the highest level (ceiling effect) ranged from $2.3 \%$ to

Table 1. Characteristics of the study population $(n=300)$

\begin{tabular}{lcc}
\hline Characteristics & $\mathrm{n}$ & $\%$ \\
\hline Age (yr) & & \\
$\quad \leq 65$ & 108 & 36 \\
$\quad>65$ & 192 & 64 \\
Sex & & \\
$\quad$ Female & 158 & 52.7 \\
$\quad$ Male & 142 & 47.3 \\
Education level & & \\
$\quad$ Illiterate & 196 & 65.3 \\
Literate & 104 & 34.7 \\
Marital Status & & \\
$\quad$ Unmarried & 69 & 77 \\
$\quad$ Married & 231 & 23 \\
Income level (\$/mo) & & \\
$\quad \leq 170$ & 145 & 59.4 \\
$\quad>170$ & 99 & 40.6 \\
Place of residence & & 54.3 \\
$\quad$ Urban & 163 & 45.7 \\
Rural & 137 & 51.3 \\
Duration of disease (d) & & 48.7 \\
$\quad \leq 30$ & 154 & \\
$\quad>30$ & 146 & \\
\hline
\end{tabular}

$35.3 \%$, while the percentage of respondents scoring at the lowest level (floor effect) ranged from $1 \%$ to $32.3 \%$. Cronbach's alpha coefficient was applied to examine the internal consistency of the WHOQOL BREF scale (24 items) as well as its four domains. The Cronbach's alpha coefficient of the WHOQOL BREF was adequate (0.889) for all 24 questions, with the fol-

Table 3. Correlation coefficients (CC) between the two overall quality of life items (Q1 and Q2) and the four domains (DOM) of the World Health Organization Quality of Life Instrument, Short Form

\begin{tabular}{llcrrrrr}
\hline & & Q1 & Q2 & DOM 1 & DOM 2 & DOM 3 & DOM 4 \\
\hline Q1 & CC & 1 & 0.434 & 0.277 & 0.237 & 0.264 & 0.520 \\
& p-value & & $<0.001$ & $<0.001$ & $<0.001$ & $<0.001$ & $<0.001$ \\
Q2 & CC & 1 & 0.225 & 0.247 & 0.235 & 0.386 \\
& p-value & & & $<0.001$ & $<0.001$ & $<0.001$ & $<0.001$ \\
DOM 1 & CC & & & 1 & 0.373 & 0.313 & 0.365 \\
& p-value & & & $<0.001$ & $<0.001$ & $<0.001$ \\
DOM 2 & CC & & & & 1 & 0.441 & 0.480 \\
& p-value & & & & $<0.001$ & $<0.001$ \\
DOM 3 & CC & & & & 1 & 0.446 \\
& p-value & & & & & $<0.001$ \\
DOM 4 & CC & & & & & 1 \\
& p-value & & & & & \\
\hline
\end{tabular}

Table 2. Responses and missing items for each item $(n=300)$

\begin{tabular}{|c|c|c|c|c|c|}
\hline Items (n) & Missing & Mean & SD & Floor & Ceiling \\
\hline Overall QOL (1) & $0(0)$ & 3.42 & 0.92 & $13(4.3)$ & $20(6.7)$ \\
\hline Overall health (2) & $0(0)$ & 3.48 & 1.07 & $22(7.3)$ & $33(11.0)$ \\
\hline Pain (3) & $0(0)$ & 3.10 & 1.37 & $46(15.3)$ & $66(22.0)$ \\
\hline Dependence on medical aids (4) & $0(0)$ & 3.40 & 1.14 & $21(7.0)$ & $55(18.3)$ \\
\hline Positive feelings (5) & $0(0)$ & 3.11 & 1.15 & $35(11.7)$ & $36(12.0)$ \\
\hline Personal beliefs (6) & $0(0)$ & 3.40 & 1.00 & $14(4.7)$ & $48(16.0)$ \\
\hline Concentration (7) & $0(0)$ & 3.06 & 1.05 & $13(4.3)$ & $32(10.7)$ \\
\hline Security (8) & $0(0)$ & 3.82 & 0.90 & $5(1.7)$ & $70(23.3)$ \\
\hline Physical environment (9) & $0(0)$ & 3.76 & 0.92 & $3(1.0)$ & $62(20.7)$ \\
\hline Energy (10) & $0(0)$ & 2.79 & 1.01 & $24(8.0)$ & $18(6.0)$ \\
\hline Bodily image (11) & $0(0)$ & 3.67 & 0.89 & $5(1.7)$ & $61(20.3)$ \\
\hline Financial support (12) & $0(0)$ & 2.59 & 0.92 & $40(13.3)$ & $7(2.3)$ \\
\hline Accessibility of information (13) & $0(0)$ & 2.71 & 0.82 & $20(6.7)$ & $7(2.3)$ \\
\hline Leisure activities (14) & $0(0)$ & 2.09 & 0.99 & $97(32.3)$ & $5(1.7)$ \\
\hline Mobility (15) & $0(0)$ & 3.13 & 1.20 & $33(11.0)$ & $35(11.7)$ \\
\hline Sleep and rest (16) & $0(0)$ & 3.50 & 1.12 & $14(4.7)$ & $51(17.0)$ \\
\hline Activities of daily living (17) & $0(0)$ & 3.20 & 1.06 & $20(7.6)$ & $22(7.3)$ \\
\hline Work capacity (18) & $0(0)$ & 3.40 & 0.90 & $9(3.0)$ & $16(5.3)$ \\
\hline Self-esteem (19) & $0(0)$ & 3.96 & 0.90 & $4(1.3)$ & $91(30.3)$ \\
\hline Personal relationships (20) & $0(0)$ & 3.99 & 0.97 & $6(2.0)$ & $106(35.3)$ \\
\hline Sexual activity (21) & $55(18.3)$ & 3.66 & 1.03 & $10(3.3)$ & $59(19.7)$ \\
\hline Social support (22) & $0(0)$ & 3.60 & 1.07 & $16(5.3)$ & $57(19.0)$ \\
\hline Home environment (23) & $0(0)$ & 3.79 & 0.85 & $6(2.0)$ & $45(15.0)$ \\
\hline Health care (24) & $0(0)$ & 3.64 & 0.97 & $10(3.3)$ & $42(14.0)$ \\
\hline Transport (25) & $0(0)$ & 3.54 & 0.94 & $8(2.7)$ & $28(9.3)$ \\
\hline Negative feelings (26) & $0(0)$ & 3.42 & 1.37 & $33(11.0)$ & $100(33.0)$ \\
\hline
\end{tabular}

Values are presented as number (\%).

$\mathrm{SD}$, standard deviation; QOL, quality of life. 
lowing values for each domain: domain 1, 0.810; domain 2, 0.765 ; domain $3,0.731$; and domain $4,0.714$. As Table 3 shows, statistically significant correlations were found between each domain of the WHOQOL-BREF. Additionally, statistically significant correlations were observed between the overall quality of life item (Q1) and the scores of the various domains (Table 3). The paired t-test was used to compare the mean scores of the four domains of the WHOQOL-BREF. As Table 4 shows, significant differences were found between all domains of the
WHOQOL-BREF (except for the difference between domain 2 and domain 4). The total mean score of the WHOQOL-BREF was 13.19 (65.95\%). Among the different domains of the WHOQOL-BREF, the lowest and highest mean scores and percentages of responses indicating satisfaction were found for domain 1 (mean, 12.29, 51.95\%) and domain 3 (mean, 14.11, 63.20\%), respectively (Table 5). The mean score of the four domains and the total score of the WHOQOL-BREF according to the independent variables (sex, age, education level, marital status, in-

Table 4. Paired t-test for the four domains of the World Health Organization Quality of Life Instrument, Short Form

\begin{tabular}{|c|c|c|c|c|c|c|c|c|}
\hline & & \multicolumn{4}{|c|}{ Paired differences } & \multirow{3}{*}{ t-test } & \multirow{3}{*}{$d f$} & \multirow{3}{*}{$\begin{array}{c}\text { p-value } \\
\text { (two-tailed) }\end{array}$} \\
\hline & & \multirow{2}{*}{ Mean } & \multirow{2}{*}{ SD } & \multicolumn{2}{|c|}{$95 \% \mathrm{Cl}$} & & & \\
\hline & & & & Lower & Upper & & & \\
\hline Pair 1 & DOM1-DOM2 & -0.90 & 2.37 & -1.16 & -0.63 & -6.58 & 299 & $<0.001$ \\
\hline Pair 2 & DOM1-DOM3 & -1.82 & 3.38 & -2.21 & -1.44 & -9.34 & 299 & $<0.001$ \\
\hline Pair 3 & DOM1-DOM4 & -0.92 & 2.29 & -1.18 & -0.66 & -6.97 & 299 & $<0.001$ \\
\hline Pair 4 & DOM2-DOM3 & -0.92 & 3.19 & -1.29 & -0.56 & -5.01 & 299 & $<0.001$ \\
\hline Pair 5 & DOM2-DOM4 & -0.02 & 2.26 & -0.28 & 0.24 & -0.15 & 299 & 0.88 \\
\hline Pair 6 & DOM3-DOM4 & 0.90 & 3.15 & 0.55 & 1.26 & 4.97 & 299 & $<0.001$ \\
\hline
\end{tabular}

$\mathrm{SD}$, standard deviation; $\mathrm{Cl}$, confidence interval; $\mathrm{df}$, degree of freedom; DOM, domain.

Table 5. Comparison of the scores in the four domains of the World Health Organization Quality of Life Instrument, Short Form according to independent variables

\begin{tabular}{|c|c|c|c|c|c|}
\hline & Domain 1 & Domain 2 & Domain 3 & Domain 4 & Total \\
\hline Total & $12.29 \pm 1.91$ & $13.19 \pm 2.29$ & $14.11 \pm 3.45$ & $13.21 \pm 2.13$ & $13.19 \pm 1.81$ \\
\hline \multicolumn{6}{|l|}{ Age (yr) } \\
\hline$\leq 65$ & $12.84 \pm 1.83$ & $13.25 \pm 2.30$ & $14.21 \pm 3.47$ & $13.54 \pm 2.31$ & $13.46 \pm 1.87$ \\
\hline$>65$ & $11.98 \pm 1.88$ & $13.16 \pm 2.29$ & $14.06 \pm 3.45$ & $13.03 \pm 2.01$ & $13.05 \pm 1.79$ \\
\hline$p$-value & $<0.001$ & 0.73 & 0.71 & 0.05 & 0.07 \\
\hline \multicolumn{6}{|l|}{ Sex } \\
\hline Female & $12.49 \pm 1.87$ & $13.35 \pm 2.22$ & $14.70 \pm 3.09$ & $13.01 \pm 2$ & $13.39 \pm 1.73$ \\
\hline Male & $12.11 \pm 1.92$ & $13.05 \pm 2.34$ & $13.59 \pm 3.69$ & $13.39 \pm 2.24$ & $13.03 \pm 1.92$ \\
\hline$p$-value & 0.09 & 0.27 & 0.005 & 0.13 & 0.01 \\
\hline \multicolumn{6}{|c|}{ Education level } \\
\hline Illiterate & $12.04 \pm 1.87$ & $13.01 \pm 2.32$ & $13.74 \pm 3.62$ & $12.90 \pm 2.02$ & $12.92 \pm 1.84$ \\
\hline Literate & $12.76 \pm 1.88$ & $13.53 \pm 2.2$ & $14.81 \pm 3.02$ & $13.79 \pm 2.23$ & $13.72 \pm 1.72$ \\
\hline p-value & 0.002 & 0.06 & 0.007 & 0.001 & $<0.001$ \\
\hline \multicolumn{6}{|l|}{ Marital status } \\
\hline Married & $12.45 \pm 1.84$ & $13.27 \pm 2.2$ & $14.96 \pm 3.23$ & $13.33 \pm 2.04$ & $13.51 \pm 1.76$ \\
\hline Unmarried & $11.74 \pm 2.05$ & $12.91 \pm 2.58$ & $11.28 \pm 2.57$ & $12.80 \pm 2.41$ & $12.18 \pm 1.72$ \\
\hline p-value & 0.006 & 0.25 & $<0.001$ & 0.07 & $<0.001$ \\
\hline \multicolumn{6}{|c|}{ Income level (\$/mo) } \\
\hline$\leq 170$ & $12.14 \pm 1.93$ & $13.37 \pm 2.42$ & $13.87 \pm 3.91$ & $12.79 \pm 1.98$ & $13.04 \pm 1.90$ \\
\hline$>170$ & $12.60 \pm 1.99$ & $13.66 \pm 2.30$ & $14.83 \pm 3.21$ & $13.78 \pm 2.41$ & $13.71 \pm 1.88$ \\
\hline $\mathrm{p}$-value & 0.07 & 0.36 & 0.04 & 0.001 & 0.007 \\
\hline \multicolumn{6}{|c|}{ Place of residence } \\
\hline Urban & $12.24 \pm 1.92$ & $13.21 \pm 2.36$ & $14.22 \pm 3.39$ & $13.55 \pm 2.15$ & $13.31 \pm 1.85$ \\
\hline Rural & $12.35 \pm 1.90$ & $13.16 \pm 2.21$ & $13.99 \pm 3.54$ & $12.81 \pm 2.06$ & $13.08 \pm 1.82$ \\
\hline p-value & 0.62 & 0.84 & 0.56 & 0.003 & 0.28 \\
\hline \multicolumn{6}{|c|}{ Duration of disease (d) } \\
\hline$\leq 30$ & $12.03 \pm 1.83$ & $12.63 \pm 1.99$ & $13.81 \pm 3.08$ & $13.26 \pm 2.09$ & $12.93 \pm 1.66$ \\
\hline$>30$ & $12.56 \pm 1.95$ & $13.78 \pm 2.44$ & $14.43 \pm 3.79$ & $13.16 \pm 2.18$ & $13.48 \pm 1.97$ \\
\hline$p$-value & 0.02 & $<0.001$ & 0.12 & 0.68 & 0.009 \\
\hline
\end{tabular}

Values are presented as mean \pm standard deviation. 
Table 6. Reverse multiple linear regression analyses of factors significantly associated with QOL

\begin{tabular}{|c|c|c|c|c|c|c|}
\hline \multirow{2}{*}{ QOL domains } & \multirow{2}{*}{ Variables } & \multicolumn{2}{|c|}{ Unstandardized coefficients } & \multirow{2}{*}{$\begin{array}{c}\text { Standardized coefficients } \\
\text { Beta } \\
\end{array}$} & \multirow{2}{*}{$\mathrm{t}$} & \multirow{2}{*}{ p-value } \\
\hline & & Beta & SE & & & \\
\hline Domain 1 & Age & -0.699 & 0.231 & -0.176 & -3.028 & 0.003 \\
\hline Domain 2 & Duration of disease & 1.151 & 0.256 & 0.252 & 4.491 & $<0.001$ \\
\hline Domain 3 & Marital status & -4.195 & 0.468 & -0.499 & -8.968 & $<0.001$ \\
\hline Domain 4 & Income level & 0.767 & 0.306 & 0.170 & 2.507 & 0.01 \\
\hline \multirow[t]{2}{*}{ Total } & Marital status & -1.411 & 0.270 & -0.321 & -5.224 & $<0.001$ \\
\hline & Duration of disease & 0.550 & 0.230 & 0.144 & 2.388 & 0.02 \\
\hline
\end{tabular}

QOL, quality of life; SE, standard error.

come, duration of disease, and place of residence) are displayed in Table 5. As seen in Table 5, the means and percentages of responses indicating satisfaction in the total score and in domains 1, 2, 3 were lower in males than females, but this pattern was reversed in domain 4. Additionally, the means and percentages of responses indicating satisfaction were higher in all domains in married persons than in single persons (Table 5). As Table 5 illustrates, differences were found between different statuses regarding certain variables (age, sex, education level, marital status, monthly income level, place of residence, and duration of disease) in total and in the four domains of the WHOQOL at the level of significance of $\mathrm{p}<0.2$. Table 6 displays the results of reverse multiple linear regression; it is clear that duration of disease and marital status were significantly associated with the totalWHOQOL score. Age was associated with domain 1, duration of disease was associated with domain 2, marital status was associated with domain 3, and income was associated with domain 4.

\section{DISCUSSION}

In this study, we evaluated the reliability (internal consistency) of the WHOQOL-BREF questionnaire in patients with cataract. We found that WHOQOL-BREF questionnaire exhibited good internal consistency overall (Cronbach's alpha of 0.889) and that each of its domains exhibited satisfactory consistency (Cronbach's alpha $>0.7$ ). The reliability analyses of Skevington [26], Nedjat [27], Gholami [28,29], Asnani [6], and Mazaheri [12] indicated that the WHOQOL-BREF scale had an acceptable level of internal consistency (Cronbach's alpha $>0.7$ ); however, Cronbach's alpha for the social relationship domain was low $(<0.7)$ in those studies. In this study, a positive correlation between all domains of the WHOQOL-BREF was observed.All correlations were found to be statistically significant. In the Gholami [28,29] and Mazaheri [12] studies, a positive correlation between all domains of the WHOQOL-BREF was observed As shown in Table 4, the mean scores of the four domains were significantly different (except for domain 2 and domain 4), with the greatest difference observed between domain 1 and domain 3. Gholami [28,29] and Mazaheri [12] found that the mean scores of four domains were different, with the greatest difference observed between domain 1 and domain 4. In this study, among the four domains of the WHOQOL-BREF, the lowest mean satisfaction rating was found for domain 1 (physical health; mean, 12.19), implying a relatively low activity level in daily life, a greater dependence on medicinal substances and medical aids, insufficient energy and mobility, more pain and discomfort, a lack of sufficient sleep and rest, and a low capacity for work. In contrast, the highest mean score was observed in domain 3 (social relationships, 14.11), implying that the study population had relatively greater levels of satisfaction regarding their personal relationships, sexual activity, and social support. In this study, the highest SD (3.45) was observed in domain 3 (social relationships). In other studies using theWHOQOL-BREF questionnaire, it has also been observed that domain 3 had the highest SD [6,12,27-29]. The fact that the highest SD was observed in domain 3 may be associated with different interpretations of the items used in this domain as well as the small number of items.

In the present study, a multiple linear regression model demonstrated that duration of disease and marital status were significantly associated with the totalWHOQOL-BREF score, meaning that single patients and patients with a duration of disease of less than 30 days had a lower quality of life. Age was associated with domain 1 scores, and patients 65 years of age or younger had better physical health. Duration of disease was associated with domain 2 , and patients with a duration of disease of less than 30 days had poorer psychological health. Marital status was associated with domain 3, and married patients had a greater quantity of social relationships. Income level was associated with domain 4 , and patients with high income reported better environmental health. According to the results of the multiple linear regression model, different variables were associated with each of the four domains of the WHOQOL-BREF.

This study had a number of limitations. First, as this was a cross-sectional study, causality between the compared variables cannot be established. Second, the surveyed population in this 
study was relatively small.

This study showed the WHOQOL-BREF questionnaire to have good reliability in characterizing the quality of life of patients with cataract. We also found that the surveyed cataract patients had a relatively moderate quality of life. In this study, it was observed that age, marital status, monthly income level, and duration of disease were important variables influencing the quality of life of patients with cataract.

\section{ACKNOWLEDGEMENTS}

The author gratefully acknowledges the patients with cataract who willingly contributed in this study. This study was funded by the Students' Research Committee of the Neyshabur University of Medical Sciences.

\section{CONFLICT OF INTEREST}

The authors have no conflicts of interest to declare for this study.

\section{ORCID}

Ali Gholami http://orcid.org/0000-0003-0338-9368

Mahmood Tavakoli Araghi http://orcid.org/0000-0002-25492510

Fatemeh Shamsabadi http://orcid.org/0000-0001-9003-0104

Mahdiye Bayat http://orcid.org/0000-0003-3572-7878

Fatemeh Dabirkhani http://orcid.org/0000-0001-7261-1861

Farhad Moradpour http://orcid.org/0000-0002-4449-1978

Kamyar Mansori http://orcid.org/0000-0001-5008-8547

Yosef Moradi http://orcid.org/0000-0002-2936-5930

Abdolhalim Rajabi http://orcid.org/0000-0002-0698-8166

\section{REFERENCES}

1. Lee JE, Fos PJ, Zuniga MA, Kastl PR, Sung JH. Assessing healthrelated quality of life in cataract patients: the relationship between utility and health-related quality of life measurement. Qual Life Res 2000;9:1127-1135.

2. American Academy of Ophthalmology and American Society of Cataract and Refractive Surgery. White paper on cataract surgery. Ophthalmology 1996;103:1152-1156.

3. WHOQOL Group. The World Health Organization Quality of Life Assessment (WHOQOL): development and general psychometric properties. Soc Sci Med 1998;46:1569-1585.

4. Rovere H, Rossini S, Reimão R. Quality of life in patients with narcolepsy: a WHOQOL-BREF study. Arq Neuropsiquiatr 2008;66:163-
167.

5. Theofilou P. Quality of life in patients undergoing hemodialysis or peritoneal dialysis treatment. J Clin Med Res 2011;3:132-138.

6. Asnani MR, Lipps GE, Reid ME. Utility of WHOQOL-BREF in measuring quality of life in sickle cell disease. Health Qual Life Outcomes 2009;7:75.

7. Aguiar MI, Farias DR, Pinheiro ML, Chaves ES, Rolim IL, Almeida PC. Quality of life of patients that had a heart transplant: application of WHOQOL-BREF scale. Arq Bras Cardiol 2011;96:60-68.

8. Najafi M, Sheikhvatan M, Montazeri A. Quality of life-associated factors among patients undergoing coronary artery bypass surgery as measured using the WHOQOL-BREF. Cardiovasc J Afr 2009;20:284289.

9. Abdollahpour I, Salimi Y, nedjat S, jorjoran shushtari Z. Quality of life and effective factors on it among governmental staff in Boukan city. Urmia Med J 2011: 22:79 (Persian).

10. Jenaabadi H, Nastiezaie N. Quality of life of in baharan training, medical, and psychiatric research center staff in Zahedan. J Urmia Nurs Midwifery Fac 2011;9:1 (Persian).

11. Nedjat S, Montazeri A, Holakouie K, Mohammad K, Majdzadeh R. Quality of life of Tehran's population by WHOQOL-BREF questionnaire in 2005. Hakim Res J 2007;10:1-8 (Persian).

12. Mazaheri M. Overall, and specific life satisfaction domains: preliminary Iranian students norms. Iran J Public Health 2010;39:89-94.

13. Nedjat S, Montazeri A, Mohammad K, Majdzadeh R, Nabavi N, Nedjat $F$, et al. Quality of life in multiple sclerosis compared to the healthy population in Tehran. Iran J Epidemiol 2006;2:9-24 (Persian).

14. Thompson HM, Reisner SL, VanKim N, Raymond HF. Quality-oflife measurement: assessing the WHOQOL-BREF scale in a sample of high-HIV-risk transgender women in San Francisco, California. Int J Transgend 2015;16:36-48.

15. Feder K, Michaud DS, Keith SE, Voicescu SA, Marro L, Than J, et al. An assessment of quality of life using the WHOQOL-BREF among participants living in the vicinity of wind turbines. Environ Res 2015; 142:227-238.

16. Lucas-Carrasco R, Laidlaw K, Power MJ. Suitability of the WHOQOL-BREF and WHOQOL-OLD for Spanish older adults. Aging Ment Health 2011;15:595-604.

17. Van Esch L, Den Oudsten BL, De Vries J. The World Health Organization Quality of Life Instrument-Short Form (WHOQOL-BREF) in women with breast problems. Int J Clin Health Psychol 2011;11:5-22.

18. Cruz LN, Polanczyk CA, Camey SA, Hoffmann JF, Fleck MP. Quality of life in Brazil: normative values for the WHOQOL-BREF in a southern general population sample. Qual Life Res 2011;20:11231129.

19. Baumann C, Erpelding ML, Régat S, Collin JF, Briançon S. The WHOQOL-BREF questionnaire: French adult population norms for the physical health, psychological health and social relationship dimensions. Rev Epidemiol Sante Publique 2010;58:33-39.

20. Skevington SM, McCrate FM. Expecting a good quality of life in health: assessing people with diverse diseases and conditions using the WHOQOL-BREF. Health Expect 2012;15:49-62.

21. Nejat S, Montazeri A, Holakouie Naieni K, Mohammad K, Majdzadeh S. The World Health Organization quality of Life (WHOQOLBREF) questionnaire: translation and validation study of the Iranian version. J Sch Public Health Inst Public Health Res 2004;4:1-12 (Persian).

22. World Health Organization. WHOQOL-BREF: introduction, administration, scoring and generic version of the assessment; 1996 [cited 2016 Mar 3]. Available from: http://www.who.int/mental_health/media/en/76.pdf. 
23. Skevington SM, Tucker C. Designing response scales for cross-cultural use in health care: data from the development of the UK WHOQOL. Br J Med Psychol 1999;72:51-61.

24. World Health Organization. WHOQOL user manual: programme on mental health; 1998 [cited 2016 Mar 3]. Available from: http://www. who.int/mental health/evidence/who qol user manual 98.pdf.

25. Bland JM, Altman DG. Cronbach's alpha. BMJ 1997;314:572.

26. Skevington SM, Lotfy M, O'Connell KA; WHOQOL Group. The World Health Organization's WHOQOL-BREF quality of life assessment: psychometric properties and results of the international field trial. A report from the WHOQOL group. Qual Life Res 2004; 13:299-310
27. Nedjat S, Montazeri A, Holakouie K, Mohammad K, Majdzadeh R. Psychometric properties of the Iranian interview-administered version of the World Health Organization's Quality of Life Questionnaire (WHOQOL-BREF): a population-based study. BMC Health Serv Res 2008;8:61.

28. Gholami A, Azini M, Borji A, Shirazi F, Sharafi Z, Zarei E. Quality of life in patients with type 2 diabetes: application of WHOQOLBREF scale. Shiraz E Med J 2013;14:162-171.

29. Gholami A, Jahromi LM, Zarei E, Dehghan A. Application of WHOQOL-BREF in measuring quality of life in health-care staff. Int J Prev Med 2013;4:809-817. 